\title{
Evolution of spatial and energy characteristics conically divergent beams in the $\mathrm{XeF}(\mathrm{C}-\mathrm{A})$ amplifier
}

\author{
Arcadii. G. Yastremskii *a, Nikolai. G. Ivanov ${ }^{a}$, Valery. F. Losev a,b ${ }^{\text {, }}$ Yurii. N. Panchenko ${ }^{a}$ \\ ${ }^{a}$ Institute of High Current Electronics, 2/3 Akademichesky ave, SB RAS 634055, Tomsk, \\ Russia; ${ }^{b}$ National Research Tomsk Polytechnic University, 30 Lenin Ave., 634034 Tomsk, Russia.
}

\begin{abstract}
Evolution of spatial and energy characteristics start pulse with energy of $0.8 \mathrm{~mJ}$ in duration of 2 ps in the XeF(C-A) amplifier of THL-100 laser system have been studied experimentally and by numerical simulation. Laser radiation energy $\mathrm{E}=2 \mathrm{~J}$ was obtained experimentally. In that work we present the 3D-model amplification of conically diverging laser beams, that takes into account the spatial inhomogeneity of the pump, and the geometry of THL-100 laser system amplifier. Description and the test results of the model are submitted. At the start pulse energy $0.8 \mathrm{~mJ}$, the calculated radiation energy at the $\mathrm{XeF}(\mathrm{C}-\mathrm{A})$ output reaches $2.4 \mathrm{~J}$. Simulation results shows that, maximal intensity of the laser radiation in this mode reaches $\mathrm{P}=60 \mathrm{GW} / \mathrm{cm}^{2}$. The evolution of the energy and space-time structure of the laser beam in the amplifier was investigated. It is shown that in the ideal case (excluding the processes of nonlinear interaction of laser beam with an active medium), at the start pulse energy of $5 \mathrm{~mJ}$, the energy of the laser radiation at the output of the amplifier is increased to $\mathrm{E}_{\text {out }}=3.8 \mathrm{~J}$. In this mode, the maximal radiation intensity reaches a value of $\mathrm{I}=148$ $\mathrm{GW} / \mathrm{cm}^{2}$.
\end{abstract}

Keywords: THL-100 laser system, spatial inhomogeneity, numerical simulation, laser beam, radiation intensity

\section{INTRODUCTION}

Today, the amplification methods of femtosecond laser pulses with using a wide-aperture, photochemical gas amplifiers, are successfully developed ${ }^{1-6}$. In HCEI together with P.N Lebedev Physical Institute (Moskow), the powerful hybrid laser system THL-100 was created ${ }^{1}$. The laser radiation with energy $0.7 \mathrm{~J}$ and pulse length of $50 \mathrm{fs}$ (power - $14 \mathrm{TW}$ ) at wavelength $\lambda=475 \mathrm{~nm}$ was obtained ${ }^{6}$. Today it is a record value of laser radiation power in the visible wavelength range. The numerical investigations this system indicate that the kinetics of the processes in the active medium enables to increase the energy of the laser pulse to $3 \mathrm{~J}$ or more ${ }^{7-8}$. However, experimentally this mode was not realized.

It is known, that the propagation of a high-intensity laser radiation in a nonlinear medium leads to the development of small-scale self-focusing. So, one of the reasons unsuccessful attempts to increase the laser energy in the experiments may be the formation of the filaments in the active medium, which leads to damage of the mirrors. The critical power of the self-focusing of the laser radiation for an axisymmetric Gaussian beam $(\varnothing<1 \mathrm{~cm})$ on $\lambda=450 \mathrm{~nm}$ in a nitrogen reaches a few gigawatt. For large diameter beams it is interest the magnitude and the intensity distribution of the laser radiation in the cross section of the laser beam. As it will be shown below the intensity of the laser radiation in this device may reach the value more than $100 \mathrm{GW} / \mathrm{cm}^{2}$. Therefore the spatial homogeneity and the value of maximum intensity of laser radiation in this device are particularly important.

Design feature of the $\mathrm{XeF}(\mathrm{C}-\mathrm{A})$ amplifier is the pumping of the active medium through the windows on the outside surface of the laser cell that leads to the spatial inhomogeneity of the gain and to deformation of the transverse profile of the laser radiation intensity.

$\mathrm{In}^{9}$ experimentally was proved that the increasing divergence angle of the laser radiation allows significantly increasing the self-focusing threshold. Use such kind of beams also lead to reducing a spatial deformation of the laser radiation intensity. $\mathrm{In}^{10}$ the gain of such beams was theoretically investigated in the mode of a spatially homogeneous active medium. In this paper we investigate the mode when the gain is spatially inhomogeneous and it is changed during passing of a laser beam through the amplifier in the saturation mode. Influence of the gain coefficient inhomogeneity on 
the spatial structure of the laser beam intensity is not quite clear. From the general considerations, however, it can be expected that the increasing the spatial homogeneity of the gain leads to decreasing of maximum laser radiation intensity and to decreasing the likelihood of filaments formation in the amplifier.

The aim of the work is to study the impact of design features of the $\mathrm{XeF}(\mathrm{C}-\mathrm{A})$ amplifier and of the start pulse characteristics on the output energy of the laser pulse, and the study evolution of the spatial structure intensity of the laser pulse during the amplification.

\section{THL-100 LASER SYSTEM}

The hybrid femtosecond laser system of THL-100 includes a Ti-sapphire start complex, prismatic stretcher, photochemical amplifier and a glass compressor. In cross section of the active region, the amplifier has a form of regular hexagon. Pumping VUV radiation is injected in the amplifier through the windows located on the outer side. Active medium $\left(\mathrm{XeF}\left(\mathrm{C}_{0}\right)\right.$ molecules) is formed at photo dissociation of $\mathrm{XeF}_{2}$ by pumping radiation with $\lambda=170 \mathrm{~nm}$ wavelength.

The start complex generates the spectrally limited laser pulses with duration $50 \mathrm{fs}, \lambda=475 \mathrm{~nm}$ and an energy up to 20 $\mathrm{mJ}$. The pulse passes in the amplifier through the prism stretcher, where, because of negative dispersion, its duration is increased up to $2 \mathrm{ps}$.

The cross-section scheme of the active region and block of the mirrors, disposed at the input side of the $\mathrm{XeF}(\mathrm{C}-\mathrm{A})$ amplifier ${ }^{11}$ is shown in Fig. 1. A similar block of mirrors is located at the opposite side of the amplifier. Circles show the

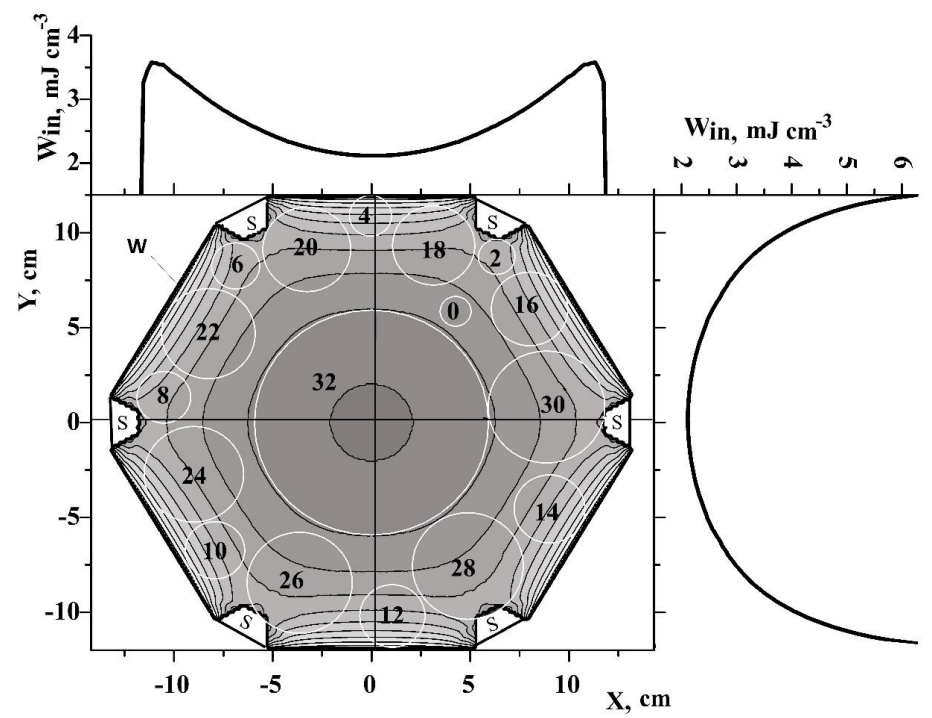

Fig. 1 Distribution of absorbed energy density in the cross section of the amplifier. Circles mirrors. The numbers in the circle - the numbers of mirrors. S - Ribs. W - Windows to input pumping radiation.

location and relative size of the mirrors. The number in the circle corresponds to passages number of the amplifier by the laser beam up to that mirror. At the near end of the amplifier the mirrors have even numbers and on the far side - odd. The start pulse enters in the amplifier through the window then it passes 33 times the amplifier active medium and exits through the window with number of 33 .

This picture also shows the calculated spatial distribution of the pump energy density, which will be discussed later. At the first 31 passages the divergence angle of the laser beam is $\Omega_{1}=1.1 \mathrm{mrad}$ and the laser pulse diameter increases from $\mathrm{D} 0=1.6 \mathrm{~cm}$ on the window of 0 to $\mathrm{D} 31=6 \mathrm{~cm}$ on the mirror of 31 . Then the divergence angle is increased to $\Omega_{2}=22$ mrad and during the subsequent passages of the active medium the laser beam diameter increases to D33 $=12 \mathrm{~cm}$ on the output window surface. To provide the maximum gain of the laser radiation, the mirrors are located near to the windows 
input of pump radiation. This location of the mirrors provides the effective start of pulse amplification. However, in this case the spatial distribution of the gain in the cross section of the laser pulse becomes non-uniform.

\section{CALCULATION METHOD}

The kinetics of reactions in gas mixture $\mathrm{N}_{2} / \mathrm{XeF}_{2}$ pumped by VUV radiation was studied quite well ${ }^{12-14}$. At the result of photolysis of $\mathrm{XeF}_{2}$ the $\mathrm{XeF}(\mathrm{BC})$ excimer molecules are formed on vibrationally excited levels. The active medium $(\mathrm{XeF}(\mathrm{B} 0, \mathrm{C} 0)$ molecules) are generated in the result of VV and VT relaxation, which determine the pump rate of the upper laser levels - $\mathrm{XeF}(\mathrm{C} 0)$. In the model $\mathrm{XeF}$ molecules are represented by the set of four effective levels: $\mathrm{XeF}(\mathrm{B})$, $\mathrm{XeF}(\mathrm{B} 0)$ - the vibrationally excited and unexcited levels of $\mathrm{XeF}(\mathrm{B})$ states; $\mathrm{XeF}(\mathrm{C}), \mathrm{XeF}(\mathrm{C} 0)$ - vibrationally excited and unexcited levels of $\mathrm{XeF}(\mathrm{C})$ states respectively. The concentrations of these molecules we will denote as $\mathrm{B}, \mathrm{B}_{0}, \mathrm{C}$ and $\mathrm{C}_{0}$ respectively.

Radial distribution of particles concentration ( $\mathrm{r}$ - the distance from the point of active medium up to the point on the surface of the window) are found by solving a system of integro-differential equations for UV radiation of pumping, and balance equations for particles concentration of the active medium ${ }^{6}$. In this paper we study the pulses whose duration ( $\tau=2.0 \mathrm{ps}$ at half maximum) is significantly shorter than the characteristic relaxation time of the vibrational states of $\mathrm{XeF}(\mathrm{B}, \mathrm{C})$. This allows us to split the time calculation of the characteristics of the active medium and modeling laser pulse amplification.

$$
\begin{gathered}
\cos (\Theta) \cdot \frac{\partial I(r, t, \Theta)}{\partial r}=-\sigma_{V U F} \cdot I(r, t, \Theta) \cdot N_{X e F_{2}} \\
\frac{\partial N_{X e F_{2}}}{\partial t}=-2 \pi N \int_{0}^{\pi} I(r, t, \Theta) \cdot \sin (\Theta) d \Theta \\
\frac{\partial B(x, y, t)}{\partial t}=\gamma_{B} W(x, y, t)-\tau_{B}^{-1} B(x, y, t)+k_{C B}^{N_{2}} C(x, y, t) \\
\frac{\partial C(x, y, t)}{\partial t}=\gamma_{C} W(x, y, t)-\tau_{C}^{-1} C(x, y, t)+k_{B C}^{N_{2}} B(x, y, t) \\
\frac{\partial B_{0}(x, y, t)}{\partial t}=\tau_{B_{v}}^{-1}\left(B_{0}^{e}(x, y, t)-B_{0}(x, y, t)\right)+k_{C_{0} B_{0}}^{N_{2}} C_{0}(x, y, t) M-\tau_{B_{0}}^{-1} B_{0}(x, y, t) \\
\frac{\partial C_{0}(x, y, t)}{\partial t}=\tau_{C_{v}}^{-1}\left(C_{0}^{e}(x, y, t)-C_{0}(x, y, t)\right)+k_{B_{0} C_{0}}^{N_{2}} B_{0}(x, y, t) M-\tau_{C_{0}}^{-1} C_{0}(x, y, t) \\
\tau_{B_{v}}^{-1}=k_{B_{v}}^{N_{2}} \cdot M, \tau_{C_{v}}^{-1}=k_{C_{v}}^{N_{2}} \cdot M
\end{gathered}
$$

Here: $\mathrm{x}$ - the horizontal distance from the center of the amplifier's cross section; $\mathrm{y}$ - the vertical distance; $C_{0}^{e}$ equilibrium concentration of molecules $\mathrm{XeF}(\mathrm{B} 0)$ and $\mathrm{XeF}(\mathrm{C} 0)$ (by the Boltzmann); $\theta$ - the angle between the direction of the pump radiation propagation and the normal to the surface of the window. I $(r, t, \theta)$ - the intensity of pump radiation; $\sigma_{\mathrm{VUF}}$ - absorption cross section of the pump radiation by molecules $\mathrm{XeF}_{2} ; \mathrm{N}, \mathrm{B}, \mathrm{C}$ and $\mathrm{N}_{2}$ concentrations of molecules $\mathrm{XeF}_{2}, \mathrm{XeF}(\mathrm{B}), \mathrm{XeF}(\mathrm{C})$ and $\mathrm{N}_{2}$, respectively; $\mathrm{W}(\mathrm{x}, \mathrm{y}, \mathrm{t})$ - number of the acts of photolysis $\mathrm{XeF}_{2}$ per unit volume per unit time in the point with coordinates $\mathrm{x}, \mathrm{y}, \mathrm{t}, k_{B_{v}}^{N_{2}}$ and $k_{C_{v}}^{N_{2}}$ - the constants of vibrational relaxation $\mathrm{B}$ and $\mathrm{C}$ states, respectively; $\gamma_{\mathrm{B}}$ and $\gamma_{\mathrm{C}}$ quantum yield of $\mathrm{XeF}(\mathrm{B})$ and $\mathrm{XeF}(\mathrm{C})$ states; $k_{B C}^{N_{2}}, k_{B_{0} C_{0}}^{N_{2}}, k_{C B}^{N_{2}}, k_{C_{0} B_{0}}^{N_{2}}$ - the rate constants of B↔C and $\mathrm{B}_{0} \leftrightarrow \mathrm{C}_{0}$ - exchange in collisions with $\mathrm{N}_{2} . Q_{B}=\sum_{i} k_{B}^{R_{i}} R_{i}$ and $Q_{C}=\sum_{i} k_{C}^{R_{i}}$ - probabilities of quenching XeF (B) and $\mathrm{XeF}(\mathrm{C})$ states by the components of mixture and by the photolysis products; $R_{i}, k_{B}^{R_{i}}, k_{C}^{R_{C}}$ - corresponding constants and reaction rates; $\sigma_{\mathrm{CA}}=9.010^{17} \mathrm{~cm}^{215}$ - stimulated emission cross section at the laser transition. The Rate coefficient used in the work are shown in Table 1. 
In this paper we are considering the amplification of expanding photons flux, the divergence angles of which are equal to $\Omega_{1}=1.1 \mathrm{mrad}$ for $1-31$ passages and $\Omega_{2}=22 \mathrm{mrad}$ for 32 and 33 passages. Angles $\Omega_{1}$ and $\Omega_{2}$ substantially greater of diffractional, therefore diffraction processes was not taken into account in the model. This makes it possible to use an algorithm of Frantz - Nodvik ${ }^{15}$.

In calculations of the gain we use the coordinate system of $\mathrm{x}_{\mathrm{L}}, \mathrm{y}_{\mathrm{L}}, \mathrm{z}_{\mathrm{L}}$ associated with the laser pulse. Here: $\mathrm{x}_{\mathrm{L}}$ and $\mathrm{x}_{\mathrm{L}}$ distances from the longitudinal axis of the flux, horizontally and vertically respectively; $Z$ - distance, traveled by the pulse in the amplifier from the input window W0. We introduce a local coordinate $\tau=t_{L}-Z / C$, where $t_{L}=t-T_{I N}-$ the propagation time of the laser pulse in the amplifier, $\mathrm{C}$ - light speed, $\mathrm{t}$ - start time of pumping the active medium, $\mathrm{T}_{\mathrm{IN}}-$ input time of start pulse into the amplifier. We introduce axis $Z_{\mathrm{L}}=\tau \cdot \mathrm{C}$, which is directed in the opposite direction with respect to the direction of axis $Z$. Point $Z_{L}=0$ corresponds to the start point of the pulse.

Table 1. Rate coefficients

\begin{tabular}{|c|c|c|}
\hline Rate coefficient & magnitude & Reference \\
\hline$k_{B}^{X e F_{2}}$ & $5.010^{-10}\left[\mathrm{~cm}^{3} \mathrm{c}^{-1}\right]$ & 16 \\
\hline$k_{C}^{X e F_{2}}$ & $2.010^{-10}\left[\mathrm{~cm}^{3} \mathrm{c}^{-1}\right]$ & 18 \\
\hline$k_{B}^{N_{2}}$ & $1.010^{-13}\left[\mathrm{~cm}^{3} \mathrm{c}^{-1}\right]$ & 18 \\
\hline$k_{C}^{N_{2}}$ & $1.010^{-13}\left[\mathrm{~cm}^{3} \mathrm{c}^{-1}\right]$ & 18 \\
\hline$k_{B C}^{N_{2}}$ & $4.410^{-11}\left[\mathrm{~cm}^{3} \mathrm{c}^{-1}\right]$ & 18 \\
\hline$\tau_{\mathrm{XeF}(\mathrm{B})}$ & $14 \quad[\mathrm{~ns}]$ & 16 \\
\hline$\tau_{\mathrm{XeF}(\mathrm{C})}$ & $100 \quad[\mathrm{~ns}]$ & 16 \\
\hline
\end{tabular}

When the beam passes through the amplifier, calculated previously distributions of the particles in the active medium $\mathrm{C} 0(\mathrm{x}, \mathrm{y}, \mathrm{t})$ are used to calculate the distribution of the gain in the cross section of the laser beam $\mathrm{C}_{01}\left(\mathrm{x}_{\mathrm{L}}, \mathrm{y}_{\mathrm{L}}, \mathrm{z}, \tau\right) * \sigma_{\mathrm{CA}}$. The wave equation for the laser photon flux $-\mathrm{F}\left(\mathrm{x}_{\mathrm{L}}, \mathrm{y}_{\mathrm{L}}, \mathrm{z}, \tau\right)$ is solved together with the equation for $\mathrm{C}_{01}\left(\mathrm{x}_{\mathrm{L}}, \mathrm{y}_{\mathrm{L}}, \mathrm{z}, \tau\right)$.

$$
\begin{aligned}
& \frac{\partial}{\partial \tau} C_{0 l}\left(x_{L}, y_{L}, z, \tau\right)=-C_{0 l}\left(x_{L}, y_{L}, z, \tau\right) \cdot \sigma_{C A} \cdot F\left(x_{L}, y_{L}, z, \tau\right) \\
& \left(\frac{\partial}{\partial Z}+K_{R}+\frac{1}{C} \frac{\partial}{\partial t}-C_{0 l}\left(x_{L}, y_{L}, z, \tau\right) \cdot \sigma_{C A}\right) \cdot F\left(x_{L}, y_{L}, z, \tau\right)=0
\end{aligned}
$$

Here: $K_{R}=2 / R$ - coefficient of laser flux attenuation due to expansion. $R$ - Curvature radius of diverging laser beam (length passage of the beam in the amplifier - $\mathrm{Z}$ plus radius curvature of the incoming beam).

Initial and boundary conditions.

$$
\begin{gathered}
F\left(x_{L}, y_{L}, z_{L}, 0\right)=F_{S T}\left(x_{L}, y_{L}, z_{L}\right) \\
C_{0 l}\left(x_{L}, y_{L}, 0, t_{L}\right)=C_{0}\left(x_{L}, y_{L}, t_{L}+t_{I N}\right)
\end{gathered}
$$

FST $\left(\mathrm{x}_{\mathrm{L}}, \mathrm{y}_{\mathrm{L}}, \mathrm{z}_{\mathrm{L}}\right)$ - the laser photons flux of the start pulse. Concentration of $C_{0 l}\left(x_{L}, y_{L}, 0, t_{L}\right)$ in the start point of the pulse is equal to the concentration of of $\mathrm{C}_{0}\left(\mathrm{x}_{\mathrm{L}}, \mathrm{y}_{\mathrm{L}}, \mathrm{t}_{\mathrm{L}}+\mathrm{t}_{\mathrm{IN}}\right)$, calculated without the laser flux.

The energy and duration of the start pulse equal to $\mathrm{E}_{\mathrm{SP}}=0.8 \mathrm{~mJ}$ and $\mathrm{T}=4 \mathrm{ps}\left(\mathrm{Z}_{\mathrm{LF}}=1.210^{-1} \mathrm{~cm}\right.$ - the length of the pulse along the $z_{L}$ axis), respectively. The photon flux distribution along $Z_{L}$ axis in start pulse is described by a Gaussian function:

$$
F_{L}(Z)=F_{0} \cdot \exp \left(-\frac{\left(Z_{L}-Z_{p i c}\right)^{2}}{Z_{p i c}^{2}} \cdot 2.77\right)
$$

Where $\mathrm{F}_{0}$ - maximal value of the photon flux in the central point $Z_{\mathrm{LPIC}}=6.0 \times 10^{-2} \mathrm{~cm}$. The beam cross section is a circle, the flux distribution along the radius in which described by the function $\mathrm{F}(\mathrm{z}, \mathrm{r})$ :

$$
F_{R}\left(z_{L}, r\right)=F_{\max }\left(z_{L}\right) \cdot \exp \left(-r^{2} \cdot 3.5\right)
$$

Here $\mathrm{r}=\left(\mathrm{x}_{\mathrm{L}}{ }^{2}+\mathrm{y}_{\mathrm{L}}^{2}\right)^{1 / 2}$. Coefficients $\mathrm{F}_{0}$ and $\mathrm{F}_{\max }\left(\mathrm{Z}_{\mathrm{L}}\right)$ were found from the normalization condition at total energy of start pulse ESP. The pulse is inputted into the amplifier through a window 0 (Figure 1) at $\mathrm{t}_{\mathrm{IN}}=80 \mathrm{~ns}$. The input time of start pulse was chosen from the condition of maximal radiation energy at the amplifier output. 
Power of the amplified spontaneous emission in this system $\mathrm{P}_{\text {ast }}<200 \mathrm{~W}^{6}$, which is substantially less the power of the laser radiation in the amplifier. Therefore, the amplification of spontaneous emission in the amplifier is not taken into account. The balance equations and the wave equation were solved by Gere method and Runge-Kutta method respectively.

Testing of the model was carried out to the different gas mixtures both before the upgrading THL-100 system ${ }^{8,19}$ (energy VUV pump radiation $E V U F=220 \mathrm{~J}$ ) and after upgrading ${ }^{20}\left(\mathrm{E}_{\mathrm{VUF}}=260 \mathrm{~J}\right)$. To testing of the model, the measured and calculated dependencies of small-signal gain vs the distance to the windows were compared for different gas mixtures $^{8,20}$. We also investigated the gain of test signal in the amplifier with a system of mirrors shown in Fig. 1. As test signal source we used the radiation of $\mathrm{cw}$ laser - Sapphire $488 \mathrm{HP}$ with a wavelength $\lambda=488 \mathrm{~nm}$ and an average power of $25 \mathrm{~mW}$. The amplification is calculated as the ratio of the radiation power in the mode of pumping, to the radiation power in mode without pumping. It was shown that the simulation data and experimental results agree well for the various pressures and gas mixtures ${ }^{7}$.

\section{RESULTS AND DISCUSSION}

The experiments and simulation were performed for gas mixture $\mathrm{N}_{2}: \mathrm{XeF}_{2}=380: 0.2$ (Torr). The pressure of $\mathrm{XeF}_{2}$ vapor was chosen from the condition of maximum energy and spatial homogeneity of the laser radiation. Pump energy, measured near the input windows surface in this mixture is equal to $E_{\mathrm{VUF}}=260 \mathrm{~J}$. Parameters of the pump pulse: duration of the pulse at the base is $300 \mathrm{~ns}$; duration of leading edge of the pulse - $100 \mathrm{~ns}$. Maximum pump radiation power $-\mathrm{P}_{\mathrm{MAX}}=1.04 \mathrm{GW}$ reached at $\mathrm{t}=100 \mathrm{~ns}$. On the output of the amplifier the laser energy $\mathrm{E}=2 \mathrm{~J}$ was obtained.

The simulation was carried out with a reflection coefficient of the mirror $\mathrm{R}=0.97$. Time dependence of the radiation power was chosen in accordance to oscillograms of pump power in a vacuum diode, and it was normalized to the total pumping energy $\mathrm{E}_{\mathrm{VUF}}$. From 260 joules of pump energy, $\mathrm{E}_{\mathrm{IN}}=157 \mathrm{~J}$ is absorbed in the active region of the amplifier. The part of pump energy is absorbed on the ribs S (Fig 1), and another part absorbed on the windows. The simulation results show that the energy of the laser radiation, in this case is equal to $2.48 \mathrm{~J}$, which agrees well with the experimental data. Decreasing of the vapor pressure down to 0.1 Torr lowers $\mathrm{E}_{\mathrm{IN}}$ down to $103 \mathrm{~J}$. The radial distribution

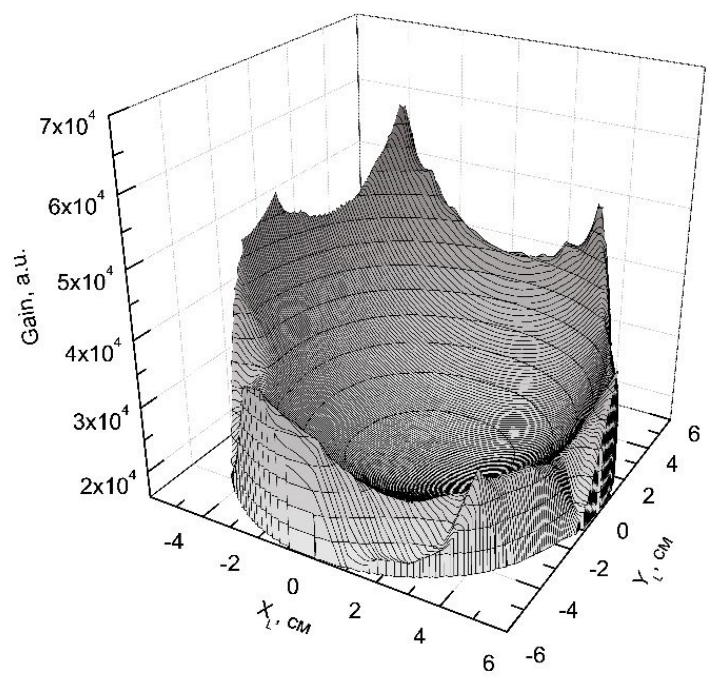

Fig. 2 Amplification in small signal gain mode. $E_{S P}=10^{-7} \mathrm{~J}$

of the energy density in this mode becomes more homogeneous but the radiation energy decreases. With increasing pressure up to 0.3 Torr the pump energy increases to $T_{\mathrm{IN}}=183 \mathrm{~J}$ and the radial distribution of pump energy density becomes more inhomogeneous.

The calculated distribution of absorbed energy density $\mathrm{W}_{\mathrm{IN}}(\mathrm{x}, \mathrm{y})$ in the cross section of the amplifier is shown in Fig.1. The maximum $\mathrm{W}_{\text {in }}=6.5 \mathrm{~mJ} / \mathrm{cm}^{3}$ achieved near the surface of input pump radiation windows. In the region of the longitudinal axis of the amplifier energy density $\mathrm{W}_{\text {in }}$ decreases to $2.2 \mathrm{~mJ} / \mathrm{cm}^{3}$. Part of pump radiation is screened by ribs 
$\mathrm{S}$, and density of absorbed energy here is reduced to $\mathrm{W}_{\text {in }}=3.6 \mathrm{~mJ} / \mathrm{cm}^{3}$. The energy of the laser radiation, in this case equals $2.48 \mathrm{~J}$, which agrees well with the experimental data.

Inhomogeneous spatial distribution of the absorbed energy and a location of the mirrors leads to the fact that, the gain of various parts of the pulse becomes dependent on its distance to the outer border of the pulse. To investigate this fact we
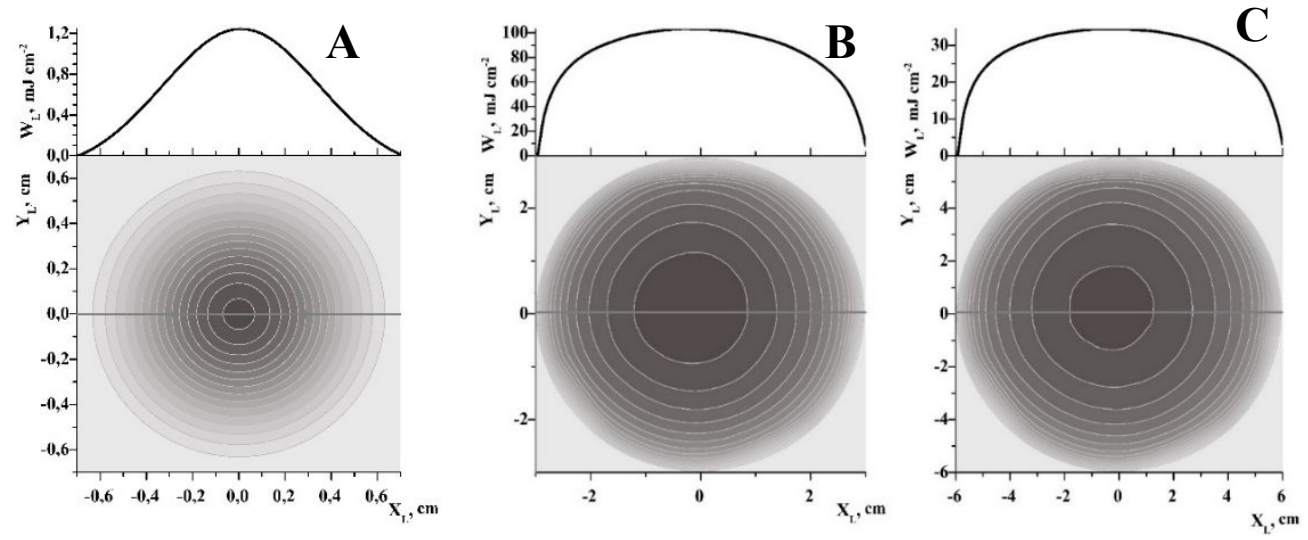

Fig. 3 Transverse distribution of laser fluence: A - Input window, B - 31 mirror. C Output window.

simulated an amplification of laser pulse with uniform distribution of energy density in the cross section in the smallsignal gain mode. Start pulse with energy density $\mathrm{W}_{\mathrm{SP}}=0.05 \cdot 10^{-6} \mathrm{~J} / \mathrm{cm}^{2}\left(\mathrm{E}_{\mathrm{SP}}=1 \cdot 10^{-7} \mathrm{~J}\right)$ was introduced in the amplifier. Distribution of the relative gain in a cross section of laser beam in small-signal gain mode is shown in Fig. 2. It can be seen, that spatial gain distribution is inhomogeneous. The maximal gain value of $7.010^{4}$ is achieved near the outer border of the beam. The minimum value of $2.0 \times 10^{4}$ is achieved on the longitudinal axis. The heterogeneities gain near the boundary are caused by the position of the mirrors with respect to the ribs S (Fig. 1). Therefore the spatial intensity distribution of the starting pulse is important. To minimize the influence of the outer border of the beam we have used a start pulse with a Gaussian radial intensity distribution of the laser radiation.

Because of design features maximum of the laser radiation density is achieved in the region of 31 mirror. Then the laser beam expands and laser radiation density decreases. Fig 3 shows the laser energy distribution in different points of the amplifier: A - on the Input window, B - on the surface of 31 mirror, C - on the output window. Distribution of the laser radiation density weakly dependent on azimuthal angle and it has a maximum in center region. Near the 31 mirror the maximum laser radiation density value is $\mathrm{W}_{\mathrm{L}}=100 \mathrm{~mJ} / \mathrm{cm}^{2}$. On the output window, $\mathrm{W}_{\mathrm{L}}$ is reduced to $32 \mathrm{~mJ} / \mathrm{cm}^{2}$. The Gaussian distribution of energy density $\mathrm{W}(\mathrm{r})=\mathrm{C} \exp \left\{-\mathrm{r}^{2}\right\}$ at the input to amplifier during the amplification transforms to distribution "close" to Super Gaussian W(r) $=\mathrm{C} \exp \left\{-\mathrm{r}^{4}\right\}$.
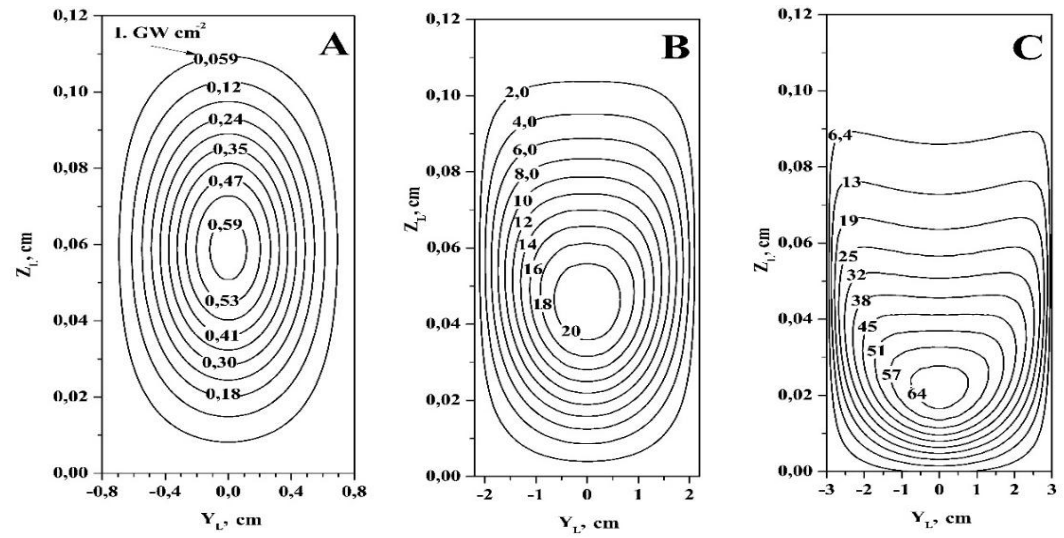

Fig.4 Distribution of the laser radiation intensity in the $Y_{L} Z_{L}$ plane of the beam in various points of the amplifier: A - 1 pass through amplifier (mirror 1), B - 20 of passages through amplifier (mirror 20), C - 31 of passes through amplifier (mirror 31 ). 
The particular interest is the evolution in the spatial intensity distribution of the laser radiation at different points of the amplifier. The contours equal-intensity of the laser radiation in the $\mathrm{Y}_{\mathrm{L}} \mathrm{Z}_{\mathrm{L}}$ plane cross section of the beam is shown on Fig.4 in various points of the amplifier: A - one pass through the amplifier (mirror 1), B - 20 passes (mirror 20), C - 31 passes (mirror 31).

After the first passage of the amplifier active region the maximum intensity achieves a value $\mathrm{I}=0.6 \mathrm{GW} / \mathrm{cm}^{2}$ in the center of the laser beam at a distance of $Z_{L}=0.06 \mathrm{~cm}$ from the start point of the pulse. After 20 passes the maximal intensity increases up to $\mathrm{I}=21 \mathrm{GW} / \mathrm{cm}^{2}$ and the point of maximum intensity approaching to start point of the pulse

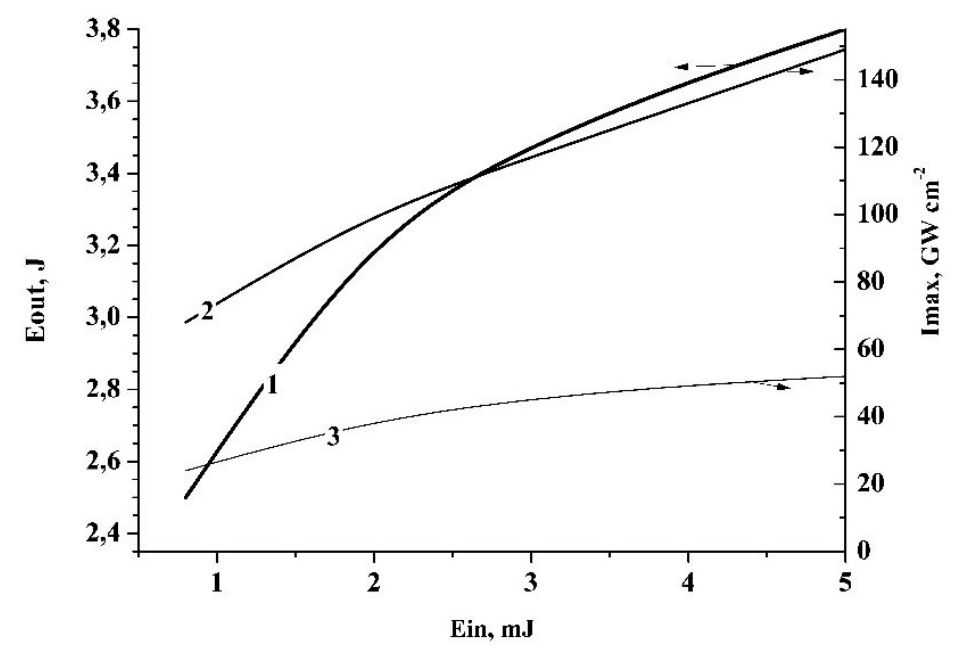

Fig 5. Laser radiation energy Eout -1, and maximum intensity Imax (mirror 31) - 2, Imax (output window 33) -3 as a functions of start pulse energy.

$\left(Z_{L}=0.04 \mathrm{~cm}\right)$. In the region of 20 mirror the pulse energy equals to $E_{L}=0.313 \mathrm{~J}$. The maximum intensity of the laser radiation $\mathrm{I}_{\mathrm{m}}=64 \mathrm{GW} \mathrm{cm}^{-2}$ is reached after 31 pass of the active medium (near the 31 mirror). The point of maximum intensity is located on the longitudinal axis of the beam at a distance $Z_{L}=0.02 \mathrm{~cm}$ from the initial point. On the distance $Z_{\mathrm{L}}>0.04 \mathrm{~cm}$ from the start point of pulse the transverse intensity distribution gets a ring shape. This behavior of the intensity is defined by the influence of the gain saturation effect on the beam axis and the heterogeneity of the gain at the beam cross section (Fig. 2).

If do not take into account the processes of nonlinear interaction of laser beam with an active medium the increased start pulse energy leads to increased energy and maximum laser radiation intensity. Fig. 5 shows: 1- the laser energy value at the output of the amplifier, 2 - the maximum intensity in the region of 31 mirror, 3 - the maximum intensity at the output of the amplifier as a functions of start pulse energy. Increasing the start pulse energy to $5 \mathrm{~mJ}$ leads to increasing laser energy to $3.8 \mathrm{~J}$. Intensity in the region of 31 mirror increases up to $148 \mathrm{GW} / \mathrm{cm}^{2}$. Point of maximum intensity in this mode is located at a distance of $\mathrm{Z}_{\mathrm{L}}=0.08 \mathrm{~cm}$ from the start point of the beam. However at the output of the amplifier the maximum intensity in this mode is not more than $50 \mathrm{GW} / \mathrm{cm}^{2}$.

\section{CONCLUSIONS}

Experimental and theoretical the amplification studies of the laser pulses in the $\mathrm{XeF}(\mathrm{C}-\mathrm{A})$ amplifier of THL-100 laser system were performed. With amplification of the start pulse with energy of $0.8 \mathrm{~mJ}$ and pulse duration of 2 ps the laser pulse energy $\mathrm{E}=2 \mathrm{~J}$ was experimentally obtained. Numerical investigations of evolution of the spatial and energy characteristics of laser beam in this mode have shown that the maximum intensity of the laser radiation reaches the value $\mathrm{Im}=64 \mathrm{GW} / \mathrm{cm}^{2}$. Moving pulse in the amplifier leads to moving the point of maximum intensity located on the longitudinal axis of the beam from the center point $\left(Z_{L}=0.06 \mathrm{~cm}\right)$ into the point $Z_{L}=0.02 \mathrm{~cm}$. The density of laser energy reaches a maximum value of $100 \mathrm{~mJ} / \mathrm{cm}^{2}$ in the center of 31 mirror. On the distance $Z_{\mathrm{L}}>0.04 \mathrm{~cm}$ from the start point of pulse the transverse intensity distribution of the laser beam takes a ring shape. This behavior of the intensity is 
defined by the influence of gain saturation effect on the beam axis and the inhomogeneity of the gain in the laser beam cross section. The Gaussian distribution of energy density $\mathrm{W}(\mathrm{r})=\mathrm{C} \exp \left\{-\mathrm{r}^{2}\right\}$ at the input to amplifier during the amplification transforms to distribution "close" to Super Gaussian W(r) $=\mathrm{C} \exp \left\{-\mathrm{r}^{4}\right\}$.

Simulation results show that if do not take into account the processes of nonlinear interaction of the laser beam with an active medium the increase of the start pulse energy to $5 \mathrm{~mJ}$ leads to increase of the laser pulse energy up to $3.8 \mathrm{~J}$. In this mode the maximum intensity reaches the value of $148 \mathrm{GW} / \mathrm{cm}^{2}$.

\section{Acknowledgements}

The experimental study was supported by the Russian Scientific Fund (Project No. 15-19-10021). The theoretical part of the work was supported by RFBR (grants №14-08-00511, 14-2802023 ОФИ-м, 13-08-00068, 13-08-98038 and 15-0802905.)

\section{REFERENCES}

[1] Aristov, A. I., Grudtsin, Ya. V., Zubarev, I. G., Ivanov, N. G., Konyashchenko, A. V., Krokhin, O. N., Losev, V. F., Mavritskii, A. O., Mamaev, S. B., Mesyats, G. A., Mikheev, L. D., Panchenko, Yu. N., Rastvortseva, A. A., Ratakhin, N. A., Sentis, M., Starodub, A. N., Tenyakov, S. Yu., Uteza, O., Tcheremiskin, V. I., Yalovoy, V. I., "Hybrid femtosecond laser system based on photochemical $\mathrm{XeF}(\mathrm{C}-\mathrm{A})$ amplifier with an aperture of $12 \mathrm{~cm}$," Atmospheric and oceanic optics 22(11), 1029-1034 (2009).

[2] Losev, V., Alekseev, S., Ivanov, N., Kovalchuk, B., Mikheev, L., Mesyats, G., Panchenko, Yu., Ratakhin, N., Yastremsky, A., "Development of a hybrid (solid state/gas) femtosecond laser system of multiterawatt peak power," Proc. SPIE Int. Soc. Eng. 7751, 09-12 (2010).

[3] Losev, V., Alekseev, S., Ivanov, N., Kovalchuk, B., Mikheev, L., Mesyats, G., Panchenko, Yu., Ratakhin, N., Yastremsky, A., "Prospects of development of hybrid (solid state/gas) ultra-high power femtosecond laser system on the basis of XeF(C-A) amplifier," Opt. Prec. Eng. 19(2), 252-259 (2011).

[4] Alekseev, S. V., Ivanov, N. G., Koval'chuk, B. M., Losev, V. F., Mesyats, G. A., Mikheev, L. D., Panchenko, Yu. N., Ratakhin, N. A., Yastremskii, A. G., "Hybrid femtosecond laser system THL-100 on the base of XeF(C-A) amplifier," Atmospheric and oceanic optics. 25(3), 221-225 (2012).

[5] Alekseev, S .V., Aristov, A. I., Ivanov, N. G., Koval'chuk, B. M., Losev, V. F., Mesyats, G. A., Mikheev, L. D., Panchenko, Yu. N., Ratakhin, N.F., "Multiterawatt femtosecond hybrid system based on a photodissociation $\mathrm{XeF}(\mathrm{C}-\mathrm{A})$ amplifier in the visible range," Quantum. Electron 42(5), 377-378 (2012).

[6] Alekseev, S, V., Aristov, A. I., Grudtsyn, Ya. V., Ivanov, N. G., Koval'chuk, B. M., Losev, V, F., Mamaev, S. B., Mesyats, G. A., Mikheev, L. D., Panchenko, Yu. N., Polivin, A. V., Stepanov, S. G., Ratakhin, N. A., Yalovoi, V. I., Yastremskii, A. G., "Visible-range hybrid femtosecond systems based on a $\mathrm{XeF}(\mathrm{C}-\mathrm{A})$ amplifier: state of the art and prospects," Quantum. Electron. 43(3), 190-200 (2013).

[7] Alekseev, S. V., Ivanov, N. G., Losev, V. F., Panchenko, Yu. N., Yastremskii, A. G., "Numerical simulation of short-pulse amplification in the active medium of $\mathrm{XeF}(\mathrm{C}-\mathrm{A})$ amplifier," Atmospheric and oceanic optics. 26(10), 863-866 (2013).

[8] Ivanov, N. G., Losev, V. F., Panchenko, Yu. N., Yastremskii, A. G., "The influence of gas mixture composition on dissipation of pumping energy in $\mathrm{XeF}(\mathrm{C}-\mathrm{A})$ amplifier of hybrid femtosecond laser system THL-100," Atmospheric and oceanic optics 27(04), 326-331 (2014).

[9] Baranova, N. B., Bykovskii, N. E., Zel'dovich, B. Ya., Senatskii, Yu. V., "Diffraction and self-focusing during amplification of high-power light pulses. II. Suppression of harmful influence of diffraction and self-focusing on a laser beam," Soviet Journal of Quantum Electronics 4(11), 1362-1366 (1975).

[10] Kuznetsova, T. I., Mikheev, L. D., "Amplification of short light pulses with a spherical wavefront," Quantum. Electron. 38(10), 969-975 (2008).

[11] Alekseev, S. V., Aristov, A. I., Ivanov, N. G., Kovalchuk, B. M., Losev, V. F., Mesyats, G. A., Mikheev, L. D., Panchenko, Yu. N., and Ratakhin, N. A., "Multiterawatt femtosecond laser system in the visible with photochemically driven XeF(C-A) boosting amplifier," Laser and Particle Beams 31(1), 17-21 (2013).

[12] Mikheev L. D., Stavrovskii, D. B., and Zuev, V. S.," Photodissociation XeF laser Operating in the visible and UV Regions," Journal of Russian Laser Research 16(5), 427 - 475 (1995). 
[13] Malinovskii, G. Ya., Mamaev, S. B., Mikheev, L. D., Moskalev, T. Yu., Sentis, M. L., Cheremiskin, V. I., Yalovoi, V. I., "Numerical simulation of the active medium and investigation of the pump source for the development of a photochemical $\mathrm{XeF}(\mathrm{C}-\mathrm{A})$ amplifier of femtosecond optical pulses," Quantum. Electron. 31(7), 617-622 (2001).

[14] Bishel, W. K., Eckstrom, D. J., Walker, H. C. and Tilton, R. A., "Photolytically pumped XeF(C-A) laser studies," J. Appl. Phys. 52(7), $4429-4434$ (1981).

[15]Frantz, L. M., Nodvik, J. S., "Theory of Pulse Propagation in a Laser Amplifier," Appl. Phys. 34(8), 234-2349, (1963).

[16] Black, G., Sharpless, R .L., Lorents, D. C., Huestis, D. L., Gutcheck, R. A., Bonifield, T. D., Helms, D. A., Walters, G. K., " $\mathrm{XeF}_{2}$ photodissociation studies. I. Quantum yields and kinetics of $\mathrm{XeF}(\mathrm{B})$ and $\mathrm{XeF}(\mathrm{C})$," J. Chem. Phys. 75(10), 4840-4846 (1981).

[17] Bibinov, N. K., Vinogradov, I. P., Mikheev, L. D., Stavrovskiĭ, D. B., "Determination of the spectral dependences of the absolute quantum yields of $\mathrm{XeF}(B, C, D)$ excimers in photolysis of $\mathrm{XeF}_{2}$,", Sov. J Quantum. Electron. 11(9), 1178-1181 (1981).

[18] Brashers, H. C., and Setser, D. W.," Transfer and quenching rate constants for $\mathrm{XeF}(\mathrm{B})$ and $\mathrm{XeF}(\mathrm{C})$ state in low vibrational levels," The J. of Chem. Phys. 76(10), 4932 - 4946 (1982).

[19]Losev, V., Alekseev, S., Ivanov, N., Kovalchuk, B., Mikheev, L., Mesyats, G., Panchenko, Yu., Puchikin A, Ratakhin, N., Yastremsky, A. "Development of a 100-trawatt hybrid femtosecond laser system," Proc. of SPIE 7993, 799317 (2011).

[20] Yastremskii, A. G., Ivanov, N. G., Losev, V. F., Panchenko, Yu. N., "Modeling of lasing possibility in XeF(C-A) amplifier of the THL-100 laser system," Proc. of SPIE 9255, 925528 (2015). 